\title{
Editorial: Oxidative Stress, Antioxidants, Transcription Factors, and Assimilation of Signal Transduction Pathways in Obesity-Related Disorders
}

\author{
David E. Stec ${ }^{1 *}$, Barbara Wegiel ${ }^{2}$ and Terry D. Hinds $\mathrm{Jr}^{3 *}$ \\ ${ }^{1}$ Department of Physiology and Biophysics, Cardiorenal and Metabolic Diseases Research Center, University of Mississippi \\ Medical Center, Jackson, MS, United States, ${ }^{2}$ Department of Surgery, Division of Surgical Oncology, Cancer Research Institute, \\ Beth Israel Deaconess Medical Center and Harvard Medical School, Boston, MA, United States, ${ }^{3}$ Department of Pharmacology \\ and Nutritional Sciences, Barnstable Brown Diabetes Center, Markey Cancer Center, University of Kentucky, Lexington, KY, \\ United States
}

Keywords: obesity, insulin resistance, therapeutics, bilirubin, reactive oxygen species, inflammation

Editorial on the Research Topic

Oxidative Stress, Antioxidants, Transcription Factors, and Assimilation of Signal Transduction Pathways in Obesity-Related Disorders

Edited and reviewed by: Salvatore Salomone,

University of Catania, Italy

*Correspondence:

David E. Stec

dstec@umc.edu

Terry D. Hinds Jr

Terry.Hinds@uky.edu

Specialty section:

This article was submitted to Experimental Pharmacology

and Drug Discovery,

a section of the journal

Frontiers in Pharmacology

Received: 16 August 2021

Accepted: 23 August 2021

Published: 07 September 2021

Citation:

Stec DE, Wegiel $B$ and Hinds Jr TD (2021) Editorial: Oxidative Stress,

Antioxidants, Transcription Factors, and Assimilation of Signal

Transduction Pathways in Obesity-

Related Disorders.

Front. Pharmacol. 12:759468.

doi: 10.3389/fphar.2021.759468
Obesity and its comorbidities such as fatty liver disease, type II diabetes, and cardiovascular disease can cause an onset of complications, worsened by the accompanying oxidative stress and deleterious signaling mechanisms, leading to irreversible damages. For instance, long-term fat accumulation in the liver may induce oxidative stress, sterile inflammation, and ultimately fibrosis, currently considered as an irreversible event. Oxidative stress occurs with an imbalance of heightened free radicals and reduced antioxidant pool in the body. Reactive oxygen species (ROS) are free oxygen-containing molecules that quickly react with other molecules within the cell leading to tissue damage. This is particularly evident in patients with obesity and metabolic disorders. Nicotinamide adenine dinucleotide phosphate (NADPH) oxidases and the mitochondrial oxidative chain complexes generate the majority of ROS in obesity and the metabolic syndrome, and this can contribute to renal dysfunction [reviewed in (Lee and Jose)]. To combat this complication, antuioxidants are the body's natural defense against ROS. They can be part of an extensive, integrated antioxidant defense system (i.e., glutathione, bilirubin) or derived from vitamins or nutritional sources such as vegetables, fruits, nuts, and seeds. Antioxidants have been proposed as potential therapies for obesity and its associated pathologies. As part of this collection of articles, Tun et al. reviewed the role of oxidative stress and the potential therapeutic role for several antioxidants in obesity and its related comorbidities (Tun et al.).

Adipose tissue is not simply a storage receptacle for excess fats that occur in obesity, but rather it is a complex endocrine organ. Adipose tissue releases hormones such as leptin and adiponectin as well as several cytokines. Hence, these are referred to as adipokines, and some are beneficial for metabolic disease (adiponectin), and others can be disadvantageous and induce inflammation (TNFa). Obesity alters the release of adipokines contributing to metabolic dysfunction and other phenotypes such as altered bone metabolism. Adiponectin levels are lower in the obese patients and may affect cementoblasts' mineralization rate, impacting periodontal tissue homeostasis and orthodontic treatment (Yong et al.). Excessive fructose consumption results in metabolic dysfunction and contributes to insulin resistance, vascular dysfunction, and hypertension. The hydrogen sulfide 
$\left(\mathrm{H}_{2} \mathrm{~S}\right)$ system is composed of enzymatic and non-enzymatic pathways capable of scavenging ROS in the cytosol and the mitochondria. Pavlovskiy et al. used $\mathrm{H}_{2} \mathrm{~S}$ donors to reduce fructose-induced gastric injury and demonstrated that they work in part by decreasing oxidative damage caused by fructose (Pavlovskiy et al.). Retinopathy is a common complication of diabetes. Hyperglycemia-induced ROS and inflammation are contributing factors to this condition. Yang et al. provided evidence that synthetic relaxin-3 (H3 relaxin) attenuated high glucose-induced nod-like receptor protein 3 (NLRP3) inflammasome activation in streptozotocin (STZ)-induced diabetic rats (Yang et al.).

Epigenetic genome modification is a tool for regulating gene expression without changes in the genomic sequence itself. Sirtuin- 6 (Sirt6) is a histone deacetylase inhibitor that controls telomere length, DNA repair, and other cellular processes. Raj et al. highlighted the emerging role of Sirt6 in the prevention of metabolic diseases and discussed the potential therapeutic use of Sirt6 modulators (Raj et al.). Histone deacetylase (HDAC) inhibitors improved fatty-acid induced insulin resistance in mice fed high fat and high fructose diets through enhanced fatty acid oxidation and improved mitochondrial function (Lee et al.).

The body produces a potent antioxidant, bilirubin, a product of heme catabolism by the enzyme, heme oxygenase-1 (HO-1), which combats ROS and oxidative stress. Mildly elevated plasma bilirubin levels correlate with reduced risks of cardiovascular and metabolic diseases (Hinds and Stec, 2018; Weaver et al., 2018; Hinds and Stec, 2019). Primary cells (podocytes and aortic endothelial cells) isolated from hyperbilirubinemic Gunn rats exhibited significantly higher viability in response to palmitic acid or angiotensin II treatment (Bianco et al.). Gunn rats also displayed sex-dependent decreases in fat mass, elevated hepatic $\beta$-oxidation, and mitochondrial function (Vidimce et al.). These studies were supported by Hana et al., who showed in this series that bilirubin treatment inhibited lipid accumulation in both skeletal muscle and liver cells and that this was dependent upon the glucose level (Hana et al.). While bilirubin is a well-known antioxidant, it might also have a hormonal function as a ligand [reviewed in (Creeden et al., 2020)] for the nuclear receptor peroxisome proliferator-activated receptor- $\alpha$ (PPAR $\alpha$ ) (Stec et al., 2016; Gordon et al., 2019; Gordon et al., 2020; Gordon et al., 2021). Hinds et al. utilized water-soluble bilirubin nanoparticles to treat obesity and its comorbidity, fatty liver disease. They showed that the bilirubin nanoparticles reduced dietaryinduced obesity and hepatic steatosis via the induction of PPARa transactivation, accelerating $\beta$-oxidation and the utilization of fat in the liver, raising plasma levels of the ketone $\beta$-hydroxybutyrate, a known hepatic fatty acid oxidation marker (Hinds et al.).

\section{REFERENCES}

Creeden, J. F., Gordon, D. M., Stec, D. E., and Hinds, T. D., Jr (2020). Bilirubin as a Metabolic Hormone: The Physiological Relevance of Low Levels. Am. J. Physiol. Endocrinol. Metab.

Gordon, D. M., Blomquist, T. M., Miruzzi, S. A., McCullumsmith, R., Stec, D. E., and Hinds, T. D., Jr (2019). RNA Sequencing in Human HepG2
Nature antioxidant remedies have been used for centuries in traditional herbal medicines like fenugreek, which treat a wide variety of ailments (Stec and Hinds, 2020). Yang et al. used 4hydroxyisoleucine, the active component of fenugreek, to prevent dietary-induced obesity inflammatory response via modification of macrophage phenotype in the adipose and liver tissues (Yang et al.). Natural products exhibit antioxidant actions through induction of pathways such as HO-1 or nuclear factor erythroid 2-related factor 2 (Nrf2) (Stec and Hinds, 2020). Kaempferol is a flavonoid compound and antioxidant found in fruits and vegetables. Yao et al. demonstrated that the vascular protective effects of kaempferol are due to its antioxidant actions via induction of Nrf2/HO-1 (Yao et al.). Bauhiniastatin-1, the active component derived from the flowering plant Bauhinia purpurea, decreased high-fat diet-induced weight gain, adiposity, and insulin resistance in rats via modulation of peroxisome proliferator-activated receptor- $\gamma(\operatorname{PPAR} \gamma)$ and AMP-activated protein kinase (AMPK) (Karunakaran et al.).

ROS and inflammation have active roles in obesity and its related pathologies. Antioxidant treatment with scavengers of ROS, cellular and mitochondrial ROS production inhibitors, or natural products that activate endogenous antioxidant pathways are potential therapies to mitigate obesity and metabolic diseases. Contributing articles to this research topic have highlighted how antioxidants may impact specific signaling pathways and transcriptional programs. Either through treatment with natural products or endogenous metabolites and hormones such as bilirubin and relaxin, targeting ROS and its associated transcription factors and signal transduction pathways offers a promise for therapy to combat obesity and its associated metabolic disorders.

\section{AUTHOR CONTRIBUTIONS}

All authors listed have made a substantial, direct, and intellectual contribution to the work and approved it for publication.

\section{FUNDING}

This work was supported by the National Institutes of Health 1R01DK121797-01A1 (TH) and 1R01DK126884-01A1 (DS), the National Heart, Lung and Blood Institute K01HL-125445 (TH) and P01 HL05197-11 (DS), and the National Institute of General Medical Sciences P20GM104357-02 (DS) and NIDDK R01 DK104714 (BW) and R01 DK125846 (BW). The content is solely the responsibility of the authors and does not necessarily represent the official views of the National Institutes of Health.

Hepatocytes Reveals PPAR-a Mediates Transcriptome Responsiveness of Bilirubin. Physiol. Genomics 51 (6), 234-240. doi:10.1152/ physiolgenomics.00028.2019

Gordon, D. M., Hong, S. H., Kipp, Z. A., and Hinds, T. D., Jr (2021). Identification of Binding Regions of Bilirubin in the Ligand-Binding Pocket of the Peroxisome ProliferatorActivated Receptor-A (PPARalpha). Molecules 26 (10). doi:10.3390/molecules 26102975 Gordon, D. M., Neifer, K. L., Hamoud, A. A., Hawk, C. F., Nestor-Kalinoski, A. L., Miruzzi, S. A., et al. (2020). Bilirubin Remodels Murine white 
Adipose Tissue by Reshaping Mitochondrial Activity and the Coregulator Profile of Peroxisome Proliferator-Activated Receptor Alpha. J. Biol. Chem.

Hinds, T. D., Jr., and Stec, D. E. (2019). Bilirubin Safeguards Cardiorenal and Metabolic Diseases: a Protective Role in Health. Curr. Hypertens. Rep. 21 (11), 87. doi:10.1007/s11906-019-0994-z

Hinds, T. D., Jr., and Stec, D. E. (2018). Bilirubin, a Cardiometabolic Signaling Molecule. Hypertension 72 (4), 788-795. doi:10.1161/ HYPERTENSIONAHA.118.11130

Stec, D. E., and Hinds, T. D., Jr. (2020). Natural Product Heme Oxygenase Inducers as Treatment for Nonalcoholic Fatty Liver Disease. Int. J. Mol. Sci. 21 (24). doi:10.3390/ijms21249493

Stec, D. E., John, K., Trabbic, C. J., Luniwal, A., Hankins, M. W., Baum, J., et al. (2016). Bilirubin Binding to PPAR $\alpha$ Inhibits Lipid Accumulation. PLoS One 11 (4), e0153427. doi:10.1371/journal.pone.0153427

Weaver, L., Hamoud, A. R., Stec, D. E., and Hinds, T. D., Jr (2018). Biliverdin Reductase and Bilirubin in Hepatic Disease. Am. J. Physiol. Gastrointest. Liver Physiol. 314 (6), G668-G76. doi:10.1152/ ajpgi.00026.2018
Conflict of Interest: DS and TH have submitted patents related to bilirubin and obesity-related disorders.

The remaining author declares that the research was conducted in the absence of any commercial or financial relationships that could be construed as a potential conflict of interest.

Publisher's Note: All claims expressed in this article are solely those of the authors and do not necessarily represent those of their affiliated organizations, or those of the publisher, the editors and the reviewers. Any product that may be evaluated in this article, or claim that may be made by its manufacturer, is not guaranteed or endorsed by the publisher.

Copyright $\odot 2021$ Stec, Wegiel and Hinds Jr. This is an open-access article distributed under the terms of the Creative Commons Attribution License (CC BY). The use, distribution or reproduction in other forums is permitted, provided the original author(s) and the copyright owner(s) are credited and that the original publication in this journal is cited, in accordance with accepted academic practice. No use, distribution or reproduction is permitted which does not comply with these terms. 